\title{
Conversational Recommender System with Explanation Facility Using Semantic Reasoning
}

\author{
Nur Rahmawati ${ }^{\# 1}$, Z.K.A Baizal ${ }^{\# 2}$, Mahmud Imrona ${ }^{\# 3}$ \\ Computational Science, Telkom University \\ Bandung, Indonesia \\ ${ }^{1}$ nurahmawati91@gmail.com \\ ${ }^{2}$ baizal@telkomuniversity.ac.id \\ ${ }_{3}^{3}$ mahmudimrona@telkomuniversity.ac.id
}

\begin{abstract}
Conversational recommender system is system that provides dialogue as user guide to obtain information from the user, in order to obtain preference for products needed. This research implements conversational recommender system with knowledge-based in the smartphone domain with an explanation facility. The recommended products are obtained based on the functional requirements of the user. Therefore, this study use ontology model as a knowledge to be more flexible in finding products that is suitable with the functional requirements of the user that is by tracing a series of semantic based on relationships in order to build the user model. We proposed an filtering method using semantic reasoning with inference method to avoid overspecialization. The evaluation result shows that the performance of our recommender system with explanation facility is more efficient than the recommendation system without explanation facility, that can be seen from the number of iterations. An interation is represented by one session of recommendation. We also notice that our system has accuracy of $84 \%$.
\end{abstract}

Keywords: recommender system, knowledge-based, ontology, semantic reasoning, inference method

\section{INTRODUCTION}

$\mathbf{T}$ HE information explosion on internet makes people difficult to find information they need immediately, particularly for on-line and e-commerce. Various kinds of products offered certainly make user difficult to decide what product he/she wants. If a user feels certain on a product he/she wants to buy, it will be easier for him/her to decide what products he/she will buy. However, if a user is still not familiar with the product or specification of the product he/she wants to buy, they will certainly find difficulties in deciding what product he/she will buy. Therefore, a system is required as to facilitate the decision making when they buy the product in accordance with the criteria they want that will also make the process efficient.

The problems can be solved by using a system helping users in a decision making, namely conversational recommender system with explanation facility. Conversational recommender system is a medium to support user in finding and choosing a product, service or information in a certain domains equipped with dialogue provided by the system as the user guide.[1]. Fernández, Y. B,et. al. implement semantic reasoning with inference method on content-based as to get the product recommendation that also uses ontology model as to handle over-specialization resulted from the implementation of content-based method with syntactic similarity [2][3]. Semantic reasoning process in this work is derived from the products previously chosen by user as to 
get recommendation for new products. It is found that the implementation of semantic reasoning with inference method can recommend various products. The use of content-based method will find some difficulties if it does not have history on the products chosen by the user.

Tintarev and Masthoff (2011) has implemented explanation facility on movie recommendation system on content based preference. Explanation facility is aimed at effectivity, trust and user's satisfaction. It is generated by using natural language based on features the user likes most. If the system does not have a complete information on the user's preference, the generation of explanation may not be optimum.

This study implements recommendation system using semantic reasoning with inference method on ontology model. However, it does not use content-based method as in the previous study, yet it uses knowledge-based method. The semantic reasoning is not also derived from the products chosen by user in the previous process, yet it is derived from the user's functional requirements. Ontology structure in this study uses structure that has been introduced by Widyantoro and Baizal (2014). The tracing process on the semantic relation comes from the products previously ranked using Multi-Attribute Utility Theory (MAUT) method that considers weight so that it is hoped that it can recommend an accurate product. The explanation facility in this study is generated by using user model built in the reasoning process by backtracking and forwarding the ontology structure using the relation among instances so the explanation result is more natural. The implementation of explanation facility in this study is aimed at improving the efficiency for users in choosing products that have been recommended.

\section{A. Definition of Ontology}

\section{ONTOLOGY}

Ontology has several definitions, Neches et.al. (1991) state that : "Ontology is a definition of a basic understanding as well as vocabulary relation in an area in accordance with the rules of combination of terms and relation for defining vocabulary". Gruber later defines ontology as an explicit specification for the terms in a domain and it also refers to the relation among terms.

From the definitions, it can be concluded that ontology is a group of terms and several specifications of the related definitions, including definition and structural relation concept in a domain as well as some possible limitations in interpreting a term.

\section{B. Ontology Components}

Ontology uses several structural variations, depending on the use of the language of ontology including the syntax used. Ontology depends on the application used, not only on the data within ontology. Ontology does not do any calculation function or other functions that processes ontology. Ontology has some components, as follows (Banowasri \& Wicaksono, 2006).

1) Concept

Concept (known as classes, object and catagories) explains concepts of a domain. A class can also have subclass which will present a concept that is more specific than that of the superclass.

2) Relation

It represents a type of interaction among concepts of a domain. Formally, it can be defined as subset of a product of $\mathrm{n}$ set, $\mathrm{R}: \mathrm{C}_{1} \times \mathrm{C}_{2} \times \ldots \times \mathrm{C}_{\mathrm{n}}$. The binary relation includes, subclass-of and connected-to, for instance. Relation should be able to define the relation of the available enitity.

3) Function

It is a specifried relation where the $n$-th element of a relation is unique for $n-1$ element. $F: C_{1} \times C_{2} \times \ldots C_{n-}$ 1 , for example Mother-of.

4) Axiom

It is used to model sentences which are always correct.

5) Instance 
Instance is the basic component of an ontology. Instance or Individual states the objects in the domain studied which is used to represent real elements comprising animals, plants, and humans, as well as abstract elements that covers numbers and letters.

\section{EXPLANATION}

Explanation in recommender system can help user in understanding the reasoning system as well as in the decision making (Billsus \& Pazzani, 1999). Based on the objective, explanation on recommender system can be divided into seven criteria (Tintarev \& Masthoff, 2011).

TABLE I

THE CRITERIA OF EXPLANATION OBJECTIVE

\begin{tabular}{|l|l|}
\hline \multicolumn{1}{|c|}{ Objective } & \multicolumn{1}{c|}{ Remarks } \\
\hline Transparency & Explaining how a system works \\
\hline Scrutability & Allowing user to correct system performance \\
\hline Trust & Increasing user's trust on system \\
\hline Effectivity & Helping user in making a precise decision \\
\hline Persuasive & Ensuring user to buy \\
\hline Efficiency & Helping user so that decision can be made immediately \\
\hline Satisfaction & Increasing convenience in the usage \\
\hline
\end{tabular}

\section{A. Multi-Attribute Utility Theory (MAUT)}

\section{FILTERING}

Multi-Attribute Utility Theory (MAUT) is an evaluation scheme to evaluate a product. Preference model derived from the MAUT theory can be represented as follow (Hu \& Aufaure, 2013),

$$
U\left(\left\langle a_{1}, a_{2}, \ldots, a_{n}\right\rangle\right)=\sum_{i=1}^{n} w_{i} V_{i}\left(a_{i}\right)
$$

where $U$ is utility of every product $\left(\left\langle a_{1}, a_{2}, \ldots, a_{n}\right\rangle\right), V_{i}$ is value function for every attribute $a_{i}$ and is relative importance of $a_{i}$. To renew user profile, utility function obtained is used. By using the attribute set $\left(x_{1}, x_{2}, \ldots, x_{m}\right)$ and relative importance of each $k_{i}$, the new value of utility is as follow:

$$
U\left(x_{1}, x_{2}, \ldots, x_{m}\right)=k_{1} U_{1}\left(x_{1}\right)+k_{2} U_{2}\left(x_{2}\right)+\ldots+k_{m} U_{m}\left(x_{m}\right)=\sum_{i=1}^{m} k_{i} U_{i}\left(x_{i}\right)
$$

where $\left(k_{1}+k_{2}+\ldots+k_{m}=1\right), 0 \leq U_{i}\left(x_{i}\right) \leq 1$ and $0 \leq U\left(x_{1}, x_{2}, \ldots, x_{m}\right) \leq 1$

\section{B. Inference Method}

Filtering process with inference method in ontology domain is conducted by taking set from property series whose individuals are in accordance with the user's preference and by determining individuals the most appropriate with the semantic intensity $\left(\lambda_{\text {Sem }}\right)$ value of the individuals (Blanco-Fernandez, et.al., 2008). The $\lambda_{\text {Sem }}$ deals with a level of relation between individuals and user's preference. Figure 1 and Figure 2 illustrate the property series user's preference as initial node. The followings are some notations in Figure 1 and Figure 2:

- $P_{U}$ refers to user'spreferences

- The number of $P_{U}$ denoted with $N_{P}$

- Each funtionality is identified as $P_{U}$ ( denoted with $x_{i}$ where $1 \leq i \leq N_{P}$ )

- The number of relation of $x_{i}$ denoted with $R_{i}$

- Each series is denoted with dinotasikan $p s_{k}^{x i}$ where $1 \leq k \leq R_{i}$

- Each $p s_{k}^{x i}$ series has $N^{k, i}$ nodes

- Node denoted with $v_{k}^{i, l}$ where $2 \leq l \leq N^{k, i}$ 
Nur RahMAWATI ET.AL.

CONVERSATional ReCOMmEnder System With...

- The priority value of user denoted with DOI (Degree of Interest)

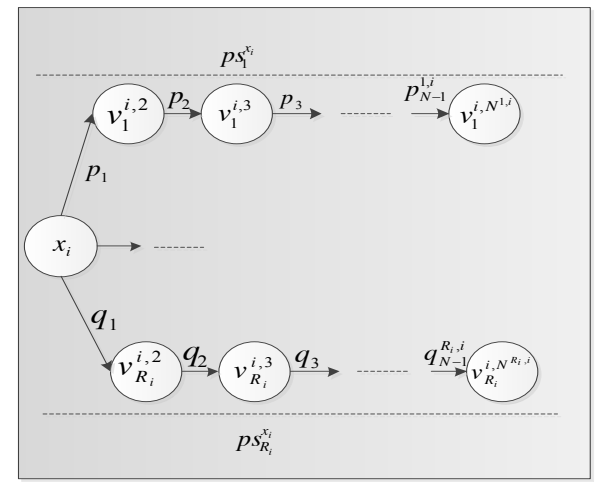

Fig. 1. Property Sequences with Initial Node $x_{i}$

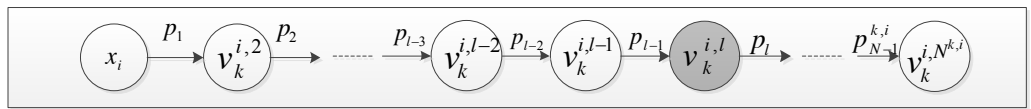

Fig. 2. Property Sequences on OWL Ontology

The $\lambda_{\text {Sem }}\left(v_{k}^{i, l}\right)$ is obtained by calculating some components :

1) User Interest Component $\left(C_{I}\right)$

$C_{I}$ is obtained by getting DOI value of user's preference in each series. The higher value of DOI at funtionality $x_{i}$ the higher $\lambda_{\text {sem }}\left(v_{k}^{i, l}\right)$ value of $v_{k}^{i, l}$ node has.

$$
C_{I}\left(v_{k}^{i}, P_{U}\right)=\operatorname{DOI}_{P_{U}}\left(x_{i}\right)
$$

2) Length of Subsequence Component $\left(C_{L}\right)$

$C_{L}$ is the parameter proper to measure the relation strenght of node origin and terminus. The longer series the smaller $C_{L}$ value. It also means that the corrrelation value is smaller.

$$
C_{L}\left(V_{k}^{i, l}, P_{U}\right)=\frac{1}{\operatorname{Length}\left(p s^{x_{i}}\right)}
$$

\section{3) Hierarchical Similarity Component $\left(C_{H}\right)$}

$C_{H}$ value is used to find the relation in hierarchy between user 's preference with instance traced.The value depends on Hierarchical Similarity $\left(\operatorname{Sim}_{\mathrm{Hie}}\right)$ between $v_{k}^{i, l}$ and instance of user preference in the same Class hierarchy, which also depends on the DOI of the instance.

The Hierarchical similarity value between $a$ and $b$ is denoted by $\left(\operatorname{Sim}_{\mathrm{Hie}}(a, b)\right)$.

where,

$$
\operatorname{Sim}_{H i e}(a, b)=\frac{\operatorname{depth}\left(L C A_{a, b}\right)}{\max (\operatorname{depth}(a), \operatorname{depth}(b))}
$$

$\operatorname{depth}(a)=$ the depth of hierarchy between root and Class of $a$.

$L C A_{a, b}=$ Class that has the lowest depth in hierarchy and it is the ancestor of Class $a$ and Class $b$

We can reformulate equation (5) as follows,

$$
M=\max \left\{\operatorname{Sim}_{H i e}\left(v_{k}^{i, l}, x_{j}\right)\right\} \forall j, 1 \leq j \leq Q
$$

where $i=$ current node 


$$
\begin{gathered}
H=\left\{x_{j} \mid \operatorname{Sim}_{H i e}\left(v_{k}^{i, l}, x_{j}\right)=M\right\} \\
C_{H}\left(v_{k}^{i, l}, P_{u}\right)=\frac{M}{|H|} \cdot \sum_{j=1}^{|H|} D O I_{P_{U}}\left(x_{j}\right), x_{j} \in H
\end{gathered}
$$

From equation (8), we can notice that the higher $\operatorname{Sim}_{H i e}$ and $x_{j}$ liked by user, the higher $C_{H}\left(v_{k}^{i, l}, P_{U}\right)$.

\section{4) Semantic Centrality Component $\left(C_{C}\right)$}

Semantic Centrality Component $\left(C_{C}\right)$ uses betweenness concept to measure the relation of nodes in a series. The betweenness concept measures the ratio of geodesic property sequence or the shortest series.

where,

$$
b_{c}(a, b)=\frac{\# g e o(a, c, b)}{\# g e o(a, b)}
$$

\# geo $(a, c, b)=$ ratio of geodesic property sequence with origin a dan terminal $b$ that involves $c$

$\# \operatorname{geo}(a, b)=$ ratio of geodesic property sequence with origin $a$ and terminal $b$

$C_{C}$ can be obtained by using the following formula,

$$
C_{C}\left(v_{k}^{i, l}, P_{u}\right)=\frac{1}{k} \sum b_{x f}\left(x_{i}, v_{k}^{i, l}\right) \cdot \frac{D O I_{P_{U}}\left(x_{f}\right)+\operatorname{DOI}\left(x_{i}\right)}{2}
$$

Note : $N_{1}=$ the number of instance on $P U$ related to $v_{k}^{i, l}$

$$
K=N_{1}\left(N_{1}-1\right)
$$

The Semantic Intensity $\left(\lambda_{S e m}, v_{k}^{i, l}\right)$ value is obtained by the following equation,

$$
\lambda_{\text {Sem }}\left(v_{k}^{i, l}\right)=w_{1} \cdot C_{1}\left(v_{k}^{i, l}, P_{U}\right) C_{L}\left(v_{k}^{i, l}, P_{U}\right)+w_{2} \cdot C_{H}\left(v_{k}^{i, l}, P_{U}\right)+w_{3} \cdot C_{C}\left(v_{k}^{i, l}, P_{U}\right)
$$

where $w_{1}=0.3, w_{2}=0.3$, and $w_{3}=0.4$

\section{A. Description of System}

\section{System Design}

Recommendation system is built with product domain of smartphone. Smartphone specification data is obtained from http://www.gsmarena.com/ and http://www.tabloidpulsa.co.id/. The data are then proceeded to be put into an ontology model which will then be used as knowledge in recommendation system which is supported by domain expert. Knowledge design is then formed into a model by using tool Protégé 4.3 .0 which will be queried with SPARQL using JENA API. Ontology model is built by making three main classes. The main class hierarchy on ontology model including the relation is as depicted on figures 3 and 4 (Widyantoro \& Baizal, 2014). 
NuR RaHMAWATI ET.AL.

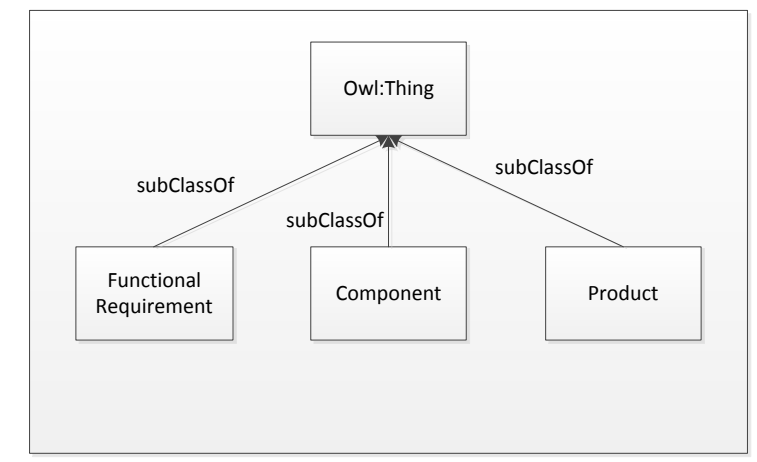

Fig. 3. The Main Classes on Ontology

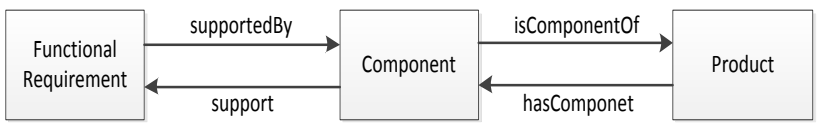

Fig. 4. Relations Among Classes

Based on Figure 3, the main class in ontology model comprises Funcional Requirement, Component, and Product. Class Functional Requirement represents functions owned by a smartphone that are probably needed by user. Class Component represents component specification of a smartphone based on the function level. While the Product class represents the smartphone products. Figure 5 depicts the sub set of the ontology.

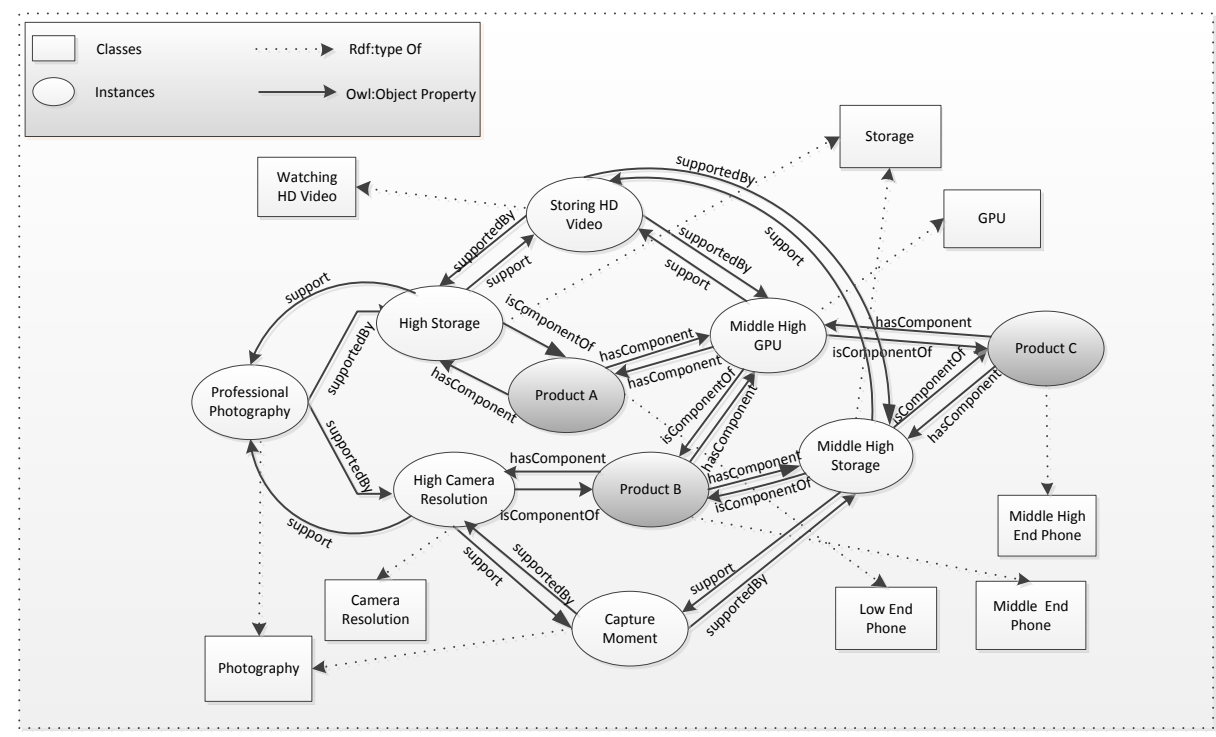

Fig. 5. Subset of Instances, Classes and Properties on Ontology.

\section{B. Filtering Process Design}

Filtering process uses semantic reasoning in tracing the series in ontology model with depth of more than two. Filtering process is a tracing of semantic relations comprising the prior top rank products, or the products resulted from MAUT. Some of products made as initials are top rank products as to generate recommendation of which hierarchy is close to the product initialization that is close in terms of semantic and functional 
requirement. Besides that, the trace at the depth of more that two can produce various products and functions so that it can prevent overspecialization.

Node tracing is conducted by using Breadth First Search (BFS) method. BFS method is exponential towards depth. Accordingly, the deeper tracing process conducted, the longer time for reasoning needed. To prevent that, limitation, in reasoning process, is conducted towards the depth of the series traced. The limitation is conducted on the level of even depth, i.e. 2, 4, 6, etc, since product node and functionality can be found on that level of depth, as can be seen on Figure 6. Therefore, it is possible if there are more various products and functionality.

Besides the limitation towards depth, to prevent excessive time needed for reasoning process, limitation is also implemented towards the value of Semantic Intensity $\left(\lambda_{\text {Sem }}\right)$ or so called threshold. Where node with $\lambda_{\text {Sem }}$ $\leq$ threshold, node will not generate their branches. It certainly will decrease the time needed for reasoning. Furthermore, the limitation for $\lambda_{\text {Sem }}$ value is conducted due to the node with $\lambda_{\text {sem }}$ below threshold ( $\lambda_{\text {Sem }}$ value is small) that makes the proximity of the node with user's preference is getting smaller.

Reasoning process is conducted by observing the node series within ontology, so that user model series are obtained. Node series within ontology consist of functionality relation, component and products illustrated in Figure 6. However, user model series obtained from reasoning are generally illustrated in Figure 7.

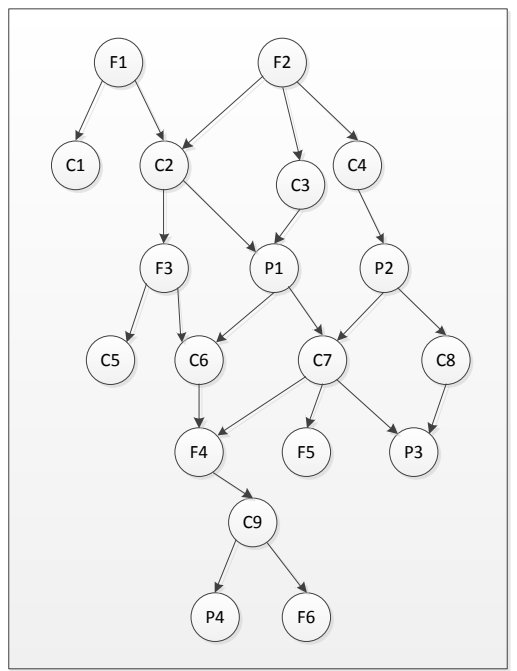

Fig. 6. Illustration on the Relation Among Functionality Nodes, Component and Product

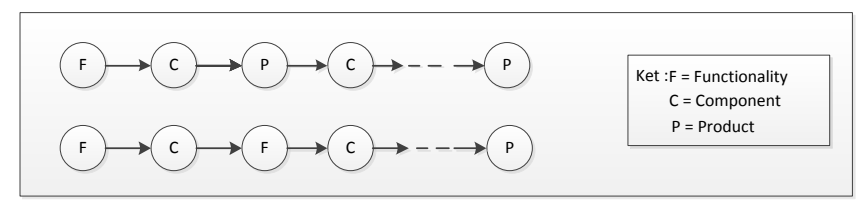

Fig. 7. The Pattern of User Model Series

In the process of reasoning, if the tracing comes to a certain node, the Semantic Intensity $\left(\lambda_{\text {Sem }}\right)$ of the node will be calculated. If the value is still above the threshold and the depth has not exceeded the limit yet, the tracing process will be continued. It may also be said that the node will generate the other nodes related to it. However, if it happens conversely, the observation may be stopped.

Semantic Intensity $\left(\lambda_{\mathrm{Sem}}\right)$ value is derived by calculating the four components, namely User Interest Component $(C I)$, Length of Subsequence Component $(C L)$, Hierarchical Similarity Component $(C H)$ and 
Nur RaHMAWATI ET.AL.

Conversational Recommender System With...

Semantic Centrality Component $(C C)$. It is this $\mathrm{CH}$ value that will be combined with the MAUT method as has been explained previously.

\section{Explanation Generation Design}

The generation of explanation on recommendation is conducted using user model generated from reasoning process. Explanation is built by reusing the available ontology structure, using explanation template (Widyantoro \& Baizal, 2014). It is conducted so that the explanation process in recommendation system is more natural as a real customer serving the customers and giving explanation pertaining to the products offered, so that user knows and understands why a product is recommended to him/her. Therefore, it will not be difficult for user to decide which product he/she will choose that can make recommending process more efficient. The explanation template is illustrated in Figure 8.

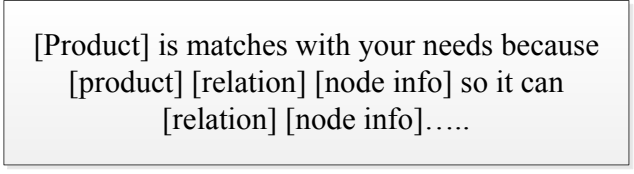

Fig. 8. Explanation Template

The generation of explanation is proceeded by tracing node of the user model obtained from semantic reasoning by backtracking or forwarding, i.e. by tracing the node of product $(P)$ through component $(C)$ and functionality $(F)$. The process is as illustrated on Figure 9.

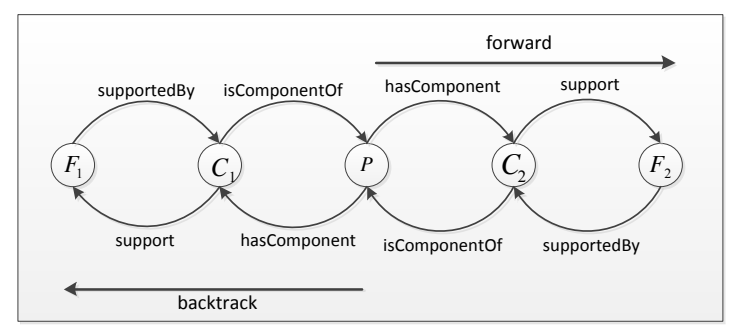

Fig. 9. Backtrack and Forward on User Model

The following is an example on the use of explanation template based on the user model in Figure 9 "Product $[P]$ matches with your need becaus product $[P]$ [hasComponent] $[C 1]$, So it can [support] $[F 1]$, and it [hasComponent] [C2] that [support] [F2]". The explanation generated depends on the number of user model generated from the reasoning process. The bigger number of user model produced, the longer explanation generated.

\section{EVALUATION}

\section{A. Evaluation Scenario}

The recommendation system test involved 50 users randomly chosen. The system testing scenario on the study comprises some research elements including time testing for the implementation of semantic reasoning with inference method in different depth, the testing on the influence of explanation facility towards the efficiency of time needed by user in making decision for the products recommended based on the number of iteration change in user preference as well as the system performance test in terms of accuracy. 


\section{B. Evaluation of Filtering Process}

The experiment was conducted by comparing time at filtering process, i.e. determining the optimum parameters obtained from some observations, namely, on the number of product initializaiton including three top products, which will be used to get the value of Hierarchical Similarity Component $(\mathrm{CH})$. Threshold and the depth of the tree used were 0.5 and 4 . The following is the comparison of filtering process in Figure 10.

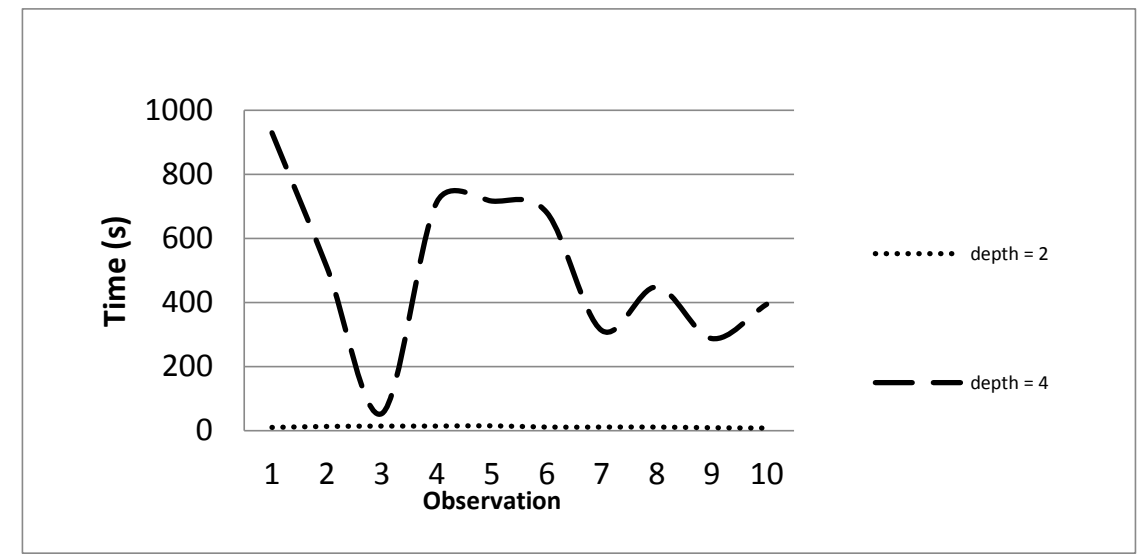

Fig. 10. Filtering Process Time at the Depth of 2 and 4

Figure 10 shows that filtering process at depth of 4 is longer than that of 2 . The observation shows that the tracing on ontology series at the depth of 4 is long enough since the BFS tracing method is exponential towards the depth, the deeper the tree traced, the longer time needed.

\section{Evaluation of the Influence of Explanation Facility}

From the test scenario of the influence of explanation facility towards the time efficiency needed by user in deciding the products recommended, the results is as in Figure11.

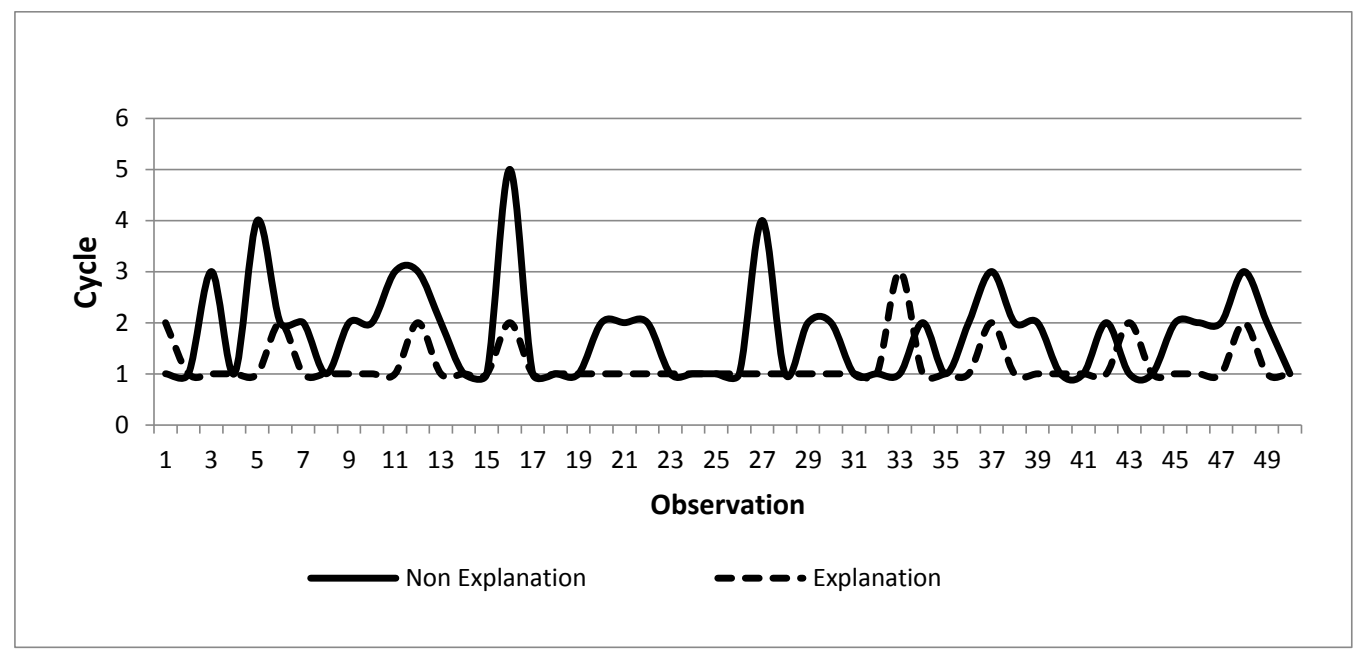

Fig.11. Comparison of the Number of Iteration 
Nur RaHMAWATI ET.AL.

Conversational Recommender System With...

The number of iteration change (cycle) in user's preference on Figure 11 shows that the number of iteration on recommendation system with explanation system is smaller that the one without explanation.

To prove and find the influence of explanation facility towards the number of iteration done by user by changing preference, $T$-test was then conducted (Supranto, 2001; Walpole, 1995).

The initial hypothesis on recommendation system by using explanation facility and the one without the facility is as follow $(\alpha=0.05)$,

$$
H_{0}: \bar{d}=0 \quad H_{1}: \bar{d}>0
$$

$\bar{d}=$ difference in the number of iteration change in user's preference on recommendation system with and without Explanation facility.

From $T$ test, it is known that $T_{\text {count }}>T_{\text {table }}(4,4772>1,6759)$, so that it can be concluded that $H_{0}$ is rejected. Therefore, it can be concluded that the number of iteration change in user's preference on recommendation system equipped with Explanation facility is less than the one withot Explanation.

TABLE II

THE ACCURACY OF RECOMMENDER SYSTEM

\begin{tabular}{|c|c|c|c|c|c|c|c|c|c|c|c|}
\hline $\begin{array}{c}\text { Domain } \\
\text { Expert }\end{array}$ & $\begin{array}{c}\mathbf{1} \\
(\boldsymbol{\%})\end{array}$ & $\begin{array}{c}\mathbf{2} \\
(\boldsymbol{\%})\end{array}$ & $\begin{array}{c}\mathbf{3} \\
(\boldsymbol{\%})\end{array}$ & $\begin{array}{c}\mathbf{4} \\
(\boldsymbol{\%})\end{array}$ & $\begin{array}{c}\mathbf{5} \\
(\boldsymbol{\%})\end{array}$ & $\begin{array}{c}\mathbf{6} \\
(\boldsymbol{\%})\end{array}$ & $\begin{array}{c}\mathbf{7} \\
(\boldsymbol{\%})\end{array}$ & $\begin{array}{c}\mathbf{8} \\
(\boldsymbol{\%})\end{array}$ & $\begin{array}{c}\mathbf{9} \\
(\boldsymbol{\%})\end{array}$ & $\begin{array}{c}\mathbf{1 0} \\
(\boldsymbol{\%})\end{array}$ & $\begin{array}{c}\text { Average } \\
(\boldsymbol{\%})\end{array}$ \\
\hline 1 & 60 & 80 & 100 & 100 & 80 & 100 & 100 & 60 & 100 & 80 & 86 \\
\hline 2 & 80 & 80 & 40 & 80 & 80 & 100 & 100 & 60 & 80 & 100 & 80 \\
\hline 3 & 80 & 80 & 100 & 100 & 100 & 40 & 100 & 60 & 100 & 100 & 86 \\
\hline \multicolumn{8}{|c|}{ Average (1,2,3)=84 \% } \\
\hline \multicolumn{10}{|c|}{}
\end{tabular}

\section{Evaluation of System Accuracy}

The system performance test in the term of accuracy was taken by using 10 scenarios done by domain expert (Cao \& Li, 2007). The accuracy level is obtained by using accuracy metrics (Olmo \& Gaudioso, 2008). The accuracy was $84 \%$, the average of testing value from three domain experts.

\section{CONCLUSION}

Based on the test and the analysis, it can be concluded that the time used by system in filtering process at an optimum parameter takes a longer time. It is because in the tree traced, the time required for filtering and semantic reasoning is longer. In addition, explanation facility features on recommendation system can increase the efficiency of time needed by user. The iteration change in preference is less in number. Meanwhile, the accuracy of recommendation system is high, as of $84 \%$.

\section{REFERENCES}

Smyth, B., McGinty L., Reilly, J., and McCarthy, K., (2004). Compound Critiques Feedback for Conversational Recommender Systems, In: The IEEE/WIC/ACM International Conference on Web Intelligence (WI-04), Beijing, China

Blanco-Fernandez, Y., Pazos-Arias, J. J., Gil-Solla, A, Ramos-Cabrer, M., Lopez-Nores, M., Garcia-Duque, J., Fernandez-Vilas, A, DiazRedondo, R. P., and Bermejo-Munoz, J., (2008). A Flexible Semantic Inference Methodology to Reason About User Preferences in Knowledge-Based Recommender Systems, Knowledge-Based Systems, vol. 21, no. 4, pp. 305-320,. 
Blanco-Fernández, Y., Pazos-Arias, J. J., Gil-Solla, A., Ramos-Cabrer, M., and Lopez-Nores, M., (2008). Providing Entertainment by Content-based Filtering and Semantic, IEEE Transactions on Consumer Electronics, vol. 54, no. 2, pp. 727-735

Tintarev, N., and Masthoff, J., (2011). Designing and evaluating explanations for recommender systems, In: Recommender Systems Handbook, F. Ricci, L. Rokach, B. Saphira dan P. B., New York: Springer US, pp. 479-510.

Widyantoro, D. H., and Baizal, Z. K. A., (2014). A Framework of Conversational Recommender System Based on User Functional Requirements, In: The 2nd IEEE Conference, International Conference on ICT.

Neches, R., Fikes, R. E., Finin, T., Gruber, T., Patil, R., Senator, T. and Swartout, W. R., (1991). Enabling Technology for Knowledge Sharing, AI MAGAZINE, vol. 12, no. 3, pp. 36-56

Banowosari, L. Y., and Wicaksana, I. W. S., (2006). Tinjauan Similaritas Semantik Dalam Pemeliharaan ontologi Pada Peer-To-Peer $(P 2 P)$, In: Komputer dan Sistem Intelejen (KOMMIT2006), Jakarta,.

Billsus, D., and Pazzani, M. J., (1999). A Personal News Agent That Talks, Learns and Explains, In: AGENTS '99 Proceedings of the third annual conference on Autonomous Agents, New York

Hu, B. dan Aufaure M., (2013). A Query Refinement Mechanism for Mobile Conversational Search in Smart Environments, In: Intelligent User Interface 2013 Workshop on Interacting with Smart Objects, Santa Monica, USA,

Supranto, J., Statistik: Teori dan Aplikasi, Jakarta: Erlangga, 2001

Walpole, R. E., (1995). Pengantar Statistika, Jakarta: Gramedia Pustaka Utama, p. 516.

Cao, Y. and Li, Y., (2007). An Intelligent Fuzzy-Based Recommendation System for Consumer Electronic Products, Expert Systems with Applications, vol. 33, no. 1, p. 230-240,.

Olmo, F. H. and Gaudioso, E., (2008). Evaluation of Recommender Systems: A New Approach, Expert Systems with Applications: An International Journal, vol. 35, no. 3, pp. 790-804, 01 October. 
